J. Phys. Chem. A

\title{
Photodissociation of Cerium Oxide Nanocluster Cations
}

\author{
S. T. Akin, ${ }^{a}$ S. G. Ard, ${ }^{a}$ B. E. Dye, ${ }^{b}$ H. F. Schaefer,,${ }^{a, b}$ and M. A. Duncan ${ }^{a} *$ \\ ${ }^{a}$ Department of Chemistry, University of Georgia, Athens, Georgia 30602, U. S. A. \\ ${ }^{\mathrm{b}}$ Center for Computational Quantum Chemistry, University of Georgia, Athens, Georgia 30602, \\ U. S. A. \\ *maduncan@uga.edu
}


$\underline{\text { Table of Contents }}$

Table $\mathrm{S} 1 . \mathrm{CeO}_{2}$ singlet relative energies, $\mathrm{D}_{\infty \mathrm{h}}-\mathrm{C}_{2 v}$, in $\mathrm{kcal} / \mathrm{mol}$ using the TZVP basis set...S4

Table S2. $\mathrm{Ce}_{2} \mathrm{O}_{3}{ }^{+}$doublet and quartet relative energies $(\mathrm{kcal} / \mathrm{mol})$ calculated at the BP86/TZVP level. The quartet geometries were started from their corresponding doublet geometries...................................................................................55

Table S3. $\mathrm{Ce}_{2} \mathrm{O}_{5}^{+}$doublet and quartet relative energies $(\mathrm{kcal} / \mathrm{mol})$ calculated at the BP86/TZVP level. The quartet geometries were started from their corresponding doublet geometries...................................................................................

Table S4. $\mathrm{Ce}_{3} \mathrm{O}_{5}^{+}$doublet and quartet relative energies $(\mathrm{kcal} / \mathrm{mol})$ calculated at the BP86/TZVP level. The quartet geometries were started from their corresponding doublet geometries.

Table $\mathrm{S5} \mathrm{Ce}_{3} \mathrm{O}_{7}^{+}$doublet and quartet relative energies $(\mathrm{kcal} / \mathrm{mol})$ calculated at the BP86/TZVP level. The quartet geometries were started from their corresponding doublet geometries.

Table S6. $\mathrm{Ce}_{4} \mathrm{O}_{7}^{+}$quartet relative energies ( $\mathrm{kcal} / \mathrm{mol}$ ) calculated at the BP86/TZVP level. The quartet geometries were started from their corresponding doublet geometries.

Table $\mathrm{S}_{7} \mathrm{Ce}_{4} \mathrm{O}_{9}^{+}$quartet relative energies (kcal/mol) calculated at the BP86/TZVP level. The quartet geometries were started from their corresponding doublet geometries.

Figure S1. Three lowest doublet and quartet isomers and optimized Cartesian coordinates in $\AA$ for $\mathrm{Ce}_{2} \mathrm{O}_{3}{ }^{+}$calculated at the BP86/TZVP level.

Figure S2. Five lowest doublet and quartet isomers and optimized Cartesian coordinates in $\AA$ for $\mathrm{Ce}_{2} \mathrm{O}_{5}{ }^{+}$calculated at the BP86/TZVP level

Figure S3. Three lowest doublet and quartet isomers and optimized Cartesian coordinates in $\AA$ for $\mathrm{Ce}_{3} \mathrm{O}_{5}{ }^{+}$calculated at the BP86/TZVP level

Figure S4. Three lowest doublet and quartet isomers and optimized Cartesian coordinates in $\AA$ for $\mathrm{Ce}_{3} \mathrm{O}_{7}^{+}$calculated at the BP86/TZVP level.

Figure S5. Four lowest doublet and quartet isomers and optimized Cartesian coordinates in $\AA$ for $\mathrm{Ce}_{4} \mathrm{O}_{7}^{+}$calculated at the BP86/TZVP level. 
Figure S6. Four lowest doublet and three lowest quartet isomers and optimized Cartesian coordinates in $\AA$ for $\mathrm{Ce}_{4} \mathrm{O}_{9}{ }^{+}$calculated at the BP86/TZVP level...........................................S28 
Table S1. $\mathrm{CeO}_{2}$ singlet relative energies, $\mathrm{D}_{\infty \mathrm{h}}-\mathrm{C}_{2 v}$, in $\mathrm{kcal} / \mathrm{mol}$ using the TZVP basis set.

\begin{tabular}{llll} 
& $\Delta \mathrm{G} 298.15 \mathrm{~K}$ & $\Delta \mathrm{H} 298.15 \mathrm{~K}$ & ZPVE \\
\hline BP86 & 5.2 & 2.3 & 3.0 \\
B3B86 & 4.4 & 1.5 & 2.2 \\
TPSS & 5.5 & 2.6 & 3.4 \\
B2PLYP & 13.6 & 10.9 & 11.6 \\
mPW2PLYP & 6.4 & 4.6 & 4.9 \\
PBE & 5.5 & 2.6 & 3.4 \\
PBE0 & 4.7 & 1.8 & 2.5 \\
MP2 & 25.9 & 21.6 & 21.9 \\
CCSD & 20.7 & 18.1 & 18.7 \\
CCSD(T) & 20.6 & 17.9 & 18.6
\end{tabular}


Table S2. $\mathrm{Ce}_{2} \mathrm{O}_{3}{ }^{+}$doublet and quartet relative energies $(\mathrm{kcal} / \mathrm{mol})$ calculated at the BP86/TZVP level. The quartet geometries were started from their corresponding doublet geometries.

\begin{tabular}{llll} 
& $\Delta \mathrm{G} 298.15 \mathrm{~K}$ & $\Delta \mathrm{H} 298.15 \mathrm{~K}$ & ZPVE \\
\hline a doublet & 0.0 & 0.0 & 0.0 \\
b doublet & 6.6 & 8.0 & 7.7 \\
c doublet & 7.8 & 9.2 & 8.9 \\
a quartet & 51.4 & 52.0 & 51.9 \\
b quartet & 69.9 & 72.2 & 71.7 \\
c quartet & 69.4 & 71.8 & 71.2
\end{tabular}


Table S3. $\mathrm{Ce}_{2} \mathrm{O}_{5}{ }^{+}$doublet and quartet relative energies ( $\mathrm{kcal} / \mathrm{mol}$ ) calculated at the BP86/TZVP level. The quartet geometries were started from their corresponding doublet geometries.

\begin{tabular}{llll} 
& $\Delta \mathrm{G} 298.15 \mathrm{~K}$ & $\Delta \mathrm{H} 298.15 \mathrm{~K}$ & ZPVE \\
\hline a doublet & 0.0 & 0.0 & 0.0 \\
b doublet & 2.4 & 3.3 & 2.9 \\
c doublet & 17.5 & 16.4 & 16.8 \\
d doublet & 49.9 & 50.8 & 50.4 \\
e doublet & 50.1 & 50.8 & 50.4 \\
a quartet & 29.4 & 32.7 & 31.9 \\
a' quartet & 27.9 & 31.5 & 30.4 \\
b quartet & 23.4 & 25.5 & 25.0 \\
c quartet & 23.4 & 25.6 & 25.1 \\
d quartet & 50.6 & 52.7 & 52.0 \\
e quartet & 51.0 & 52.7 & 52.0
\end{tabular}


Table S4. $\mathrm{Ce}_{3} \mathrm{O}_{5}{ }^{+}$doublet and quartet relative energies $(\mathrm{kcal} / \mathrm{mol})$ calculated at the BP86/TZVP level. The quartet geometries were started from their corresponding doublet geometries.

\begin{tabular}{llll} 
& $\Delta \mathrm{G} 298.15 \mathrm{~K}$ & $\Delta \mathrm{H} 298.15 \mathrm{~K}$ & ZPVE \\
\hline a doublet & 0.0 & 0.0 & 0.0 \\
b doublet & 4.1 & 6.1 & 5.6 \\
c doublet & 4.9 & 6.0 & 5.7 \\
a quartet & 53.7 & 54.6 & 54.4 \\
b quartet & 53.7 & 55.2 & 54.7 \\
c quartet & 52.3 & 54.5 & 54.0
\end{tabular}


Table S5. $\mathrm{Ce}_{3} \mathrm{O}_{7}{ }^{+}$doublet and quartet relative energies $(\mathrm{kcal} / \mathrm{mol}$ ) calculated at the BP86/TZVP level. The quartet geometries were started from their corresponding doublet geometries.

\begin{tabular}{llll} 
& $\Delta \mathrm{G} 298.15 \mathrm{~K}$ & $\Delta \mathrm{H} 298.15 \mathrm{~K}$ & ZPVE \\
\hline a doublet & 0.0 & 0.0 & 0.0 \\
b doublet & 4.9 & 5.5 & 5.5 \\
c doublet & 29.5 & 30.2 & 30.1 \\
a quartet & 26.3 & 26.3 & 26.6 \\
b quartet & 30.7 & 32.1 & 32.0 \\
c quartet & 31.3 & 32.5 & 32.4
\end{tabular}


Table S6. $\mathrm{Ce}_{4} \mathrm{O}_{7}^{+}$quartet relative energies ( $\left.\mathrm{kcal} / \mathrm{mol}\right)$ calculated at the BP86/TZVP level. The quartet geometries were started from their corresponding doublet geometries.

\begin{tabular}{llll} 
& $\Delta \mathrm{G} 298.15 \mathrm{~K}$ & $\Delta \mathrm{H} 298.15 \mathrm{~K}$ & ZPVE \\
\hline a doublet & 0.0 & 0.0 & 0.0 \\
b doublet & 0.3 & 0.4 & 0.4 \\
c doublet & 7.0 & 7.5 & 7.2 \\
d doublet & 17.2 & 17.1 & 16.7 \\
a quartet & 52.2 & 54.3 & 53.7 \\
b quartet & 57.1 & 58.6 & 58.4 \\
c quartet & 57.1 & 58.7 & 58.5 \\
d quartet & 68.1 & 69.8 & 69.5
\end{tabular}


Table S7. $\mathrm{Ce}_{4} \mathrm{O}_{9}{ }^{+}$quartet relative energies $(\mathrm{kcal} / \mathrm{mol})$ calculated at the BP86/TZVP level. The quartet geometries were started from their corresponding doublet geometries.

\begin{tabular}{llll} 
& $\Delta \mathrm{G} 298.15 \mathrm{~K}$ & $\Delta \mathrm{H} 298.15 \mathrm{~K}$ & ZPVE \\
\hline a doublet & 0.0 & 0.0 & 0.0 \\
b doublet & 1.5 & 1.6 & 1.4 \\
c doublet & 1.8 & 3.1 & 2.3 \\
d doublet & 2.6 & 3.4 & 3.2 \\
a quartet & 25.2 & 26.9 & 26.5 \\
b quartet & 24.8 & 26.8 & 26.4 \\
c quartet & 28.1 & 31.9 & 30.7
\end{tabular}



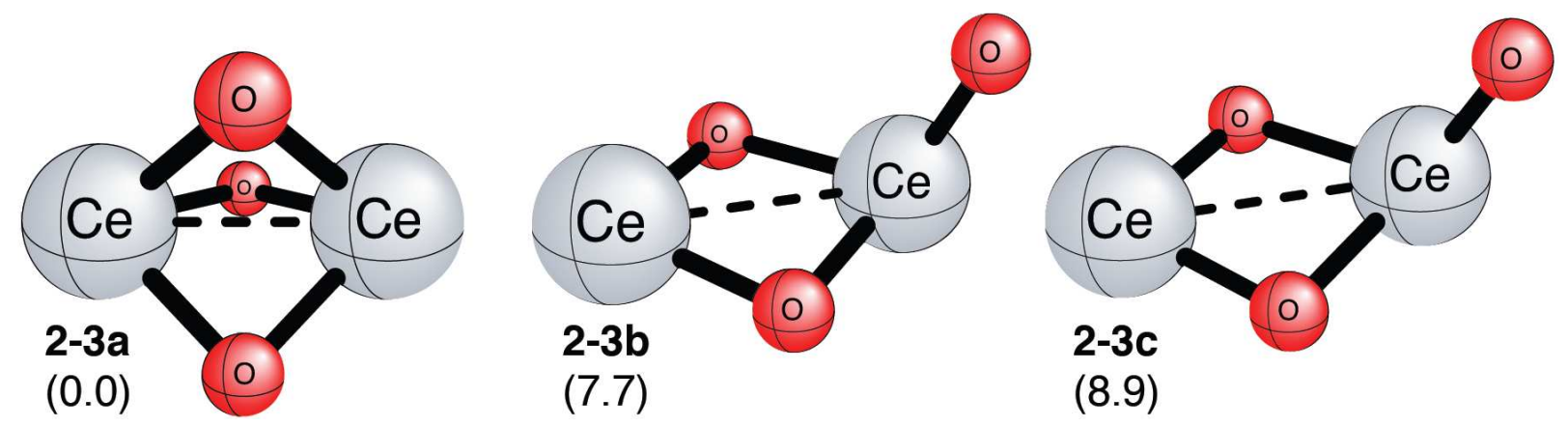

Figure S1. Three lowest isomers of $\mathrm{Ce}_{2} \mathrm{O}_{3}{ }^{+}$calculated at the BP86/TZVP level.

Although, the global minimum has pseudo $\mathrm{D}_{3 h}$ symmetry, NBO analysis shows that one Ce has a double bond to each $\mathrm{O}$ and the other $\mathrm{Ce}$ has only a single bond to each $\mathrm{O}$. A half filled Ce-Ce bond is also found. There are two geometrically similar low-lying isomers within 9 $\mathrm{kcal} / \mathrm{mol}$ of the global minimum. These isomers are formed by increasing the $89.2^{\circ} \mathrm{O}-\mathrm{Ce}-\mathrm{Ce}$ angle of the global minimum. The lower lying isomer, $7.7 \mathrm{kcal} / \mathrm{mol}$, has an O-Ce-Ce angle of $104.2^{\circ}$ and the higher lying isomer, $8.9 \mathrm{kcal} / \mathrm{mol}$, has an angle of $108.3^{\circ}$. As the $\mathrm{O}$ swings away from one Ce it contracts towards the other Ce, changing the bond length from $2.10 \AA$ to $1.80 \AA$. With the reduction in symmetric bonding the $2.96 \AA \mathrm{Ce}-\mathrm{Ce}$ bond increases by $0.38 \AA$ and $0.40 \AA$ for the $7.7 \mathrm{kcal} / \mathrm{mol}$ and $8.9 \mathrm{kcal} / \mathrm{mol}$ isomers respectively. Population in the $\mathrm{Ce}-\mathrm{Ce}$ bond is only reduced from 0.96 electrons to 0.95 electrons for the $7.7 \mathrm{kcal} / \mathrm{mol}$ isomer but the population is reduced to 0.84 electrons for the $8.9 \mathrm{kcal} / \mathrm{mol}$ isomer.

Optimized Cartesian coordinates in $\AA$ for lowest doublet and quartet isomers of $\mathrm{Ce}_{2} \mathrm{O}_{3}{ }^{+}$ calculated at the BP86/TZVP level.

a doublet

$\begin{array}{lrrr}\mathrm{Ce} & -0.000575 & 0.000839 & 1.472423 \\ \mathrm{O} & -1.484655 & -0.152929 & -0.000252 \\ \mathrm{O} & 0.611516 & 1.362230 & 0.000424 \\ \mathrm{O} & 0.873818 & -1.210992 & 0.000429 \\ \mathrm{Ce} & -0.000438 & 0.000853 & -1.473030\end{array}$


b doublet

$\begin{array}{lrrr}\mathrm{O} & 0.613261 & 0.053111 & 2.055106 \\ \mathrm{Ce} & -0.795730 & -0.061116 & 0.938995 \\ \mathrm{Ce} & 0.557225 & 0.039569 & -2.098177 \\ \mathrm{O} & -0.086317 & -1.309238 & -0.775898 \\ \mathrm{O} & -0.289020 & 1.277672 & -0.781788\end{array}$

c doublet

$\begin{array}{lrrr}\mathrm{O} & 0.589075 & 0.001302 & 2.923567 \\ \mathrm{Ce} & -0.769984 & -0.003107 & 1.742886 \\ \mathrm{O} & -0.172754 & 1.293031 & 0.012890 \\ \mathrm{O} & -0.167253 & -1.293542 & 0.011606 \\ \mathrm{Ce} & 0.520915 & 0.002317 & -1.342590\end{array}$

a quartet

$\begin{array}{lrrr}\mathrm{Ce} & -0.025473 & 0.136230 & 1.565283 \\ \mathrm{O} & -0.285603 & 1.522113 & -0.000680 \\ \mathrm{O} & 1.287957 & -0.690378 & 0.000175 \\ \mathrm{O} & -0.957915 & -1.103735 & 0.002069 \\ \mathrm{Ce} & -0.021602 & 0.135769 & -1.564088\end{array}$

b quartet

$\begin{array}{lrrr}\mathrm{O} & 0.626141 & 0.097194 & 2.279466 \\ \mathrm{Ce} & -0.785974 & -0.091010 & 0.866612 \\ \mathrm{Ce} & 0.486272 & 0.044014 & -2.207894 \\ \mathrm{O} & -0.084924 & -1.337297 & -0.819349 \\ \mathrm{O} & -0.242096 & 1.287097 & -0.780596\end{array}$

c quartet

$\begin{array}{lrrr}\mathrm{O} & 0.231089 & 0.003682 & 3.549936 \\ \mathrm{Ce} & -0.227675 & -0.004822 & 1.649618 \\ \mathrm{O} & -0.052885 & 1.314957 & -0.091940 \\ \mathrm{O} & -0.051788 & -1.316807 & -0.095383 \\ \mathrm{Ce} & 0.101257 & 0.002991 & -1.663872\end{array}$




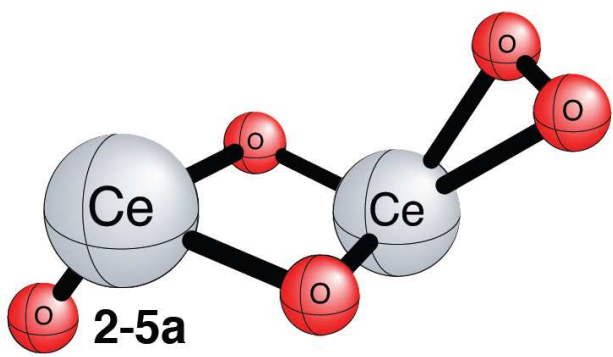

$(0.0)$

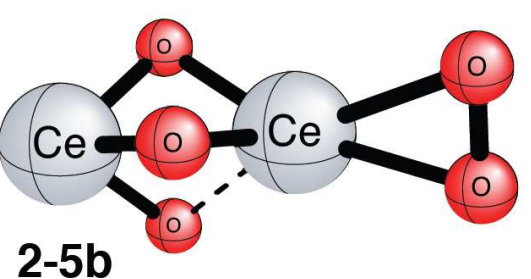

(2.9)

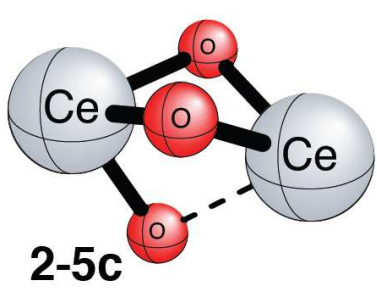

(16.8)

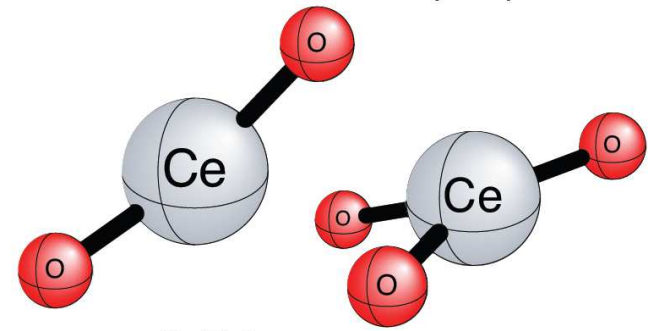

2-5d $(50.4)$

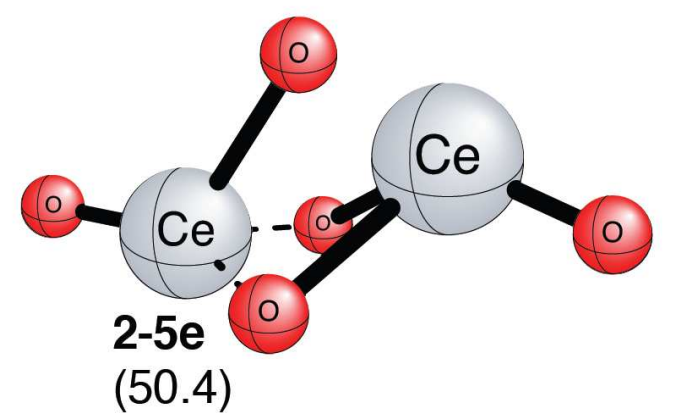

Figure S2. Five lowest isomers of $\mathrm{Ce}_{2} \mathrm{O}_{5}{ }^{+}$calculated at the BP86/TZVP level.

The global minimum is formed with the addition of $\mathrm{O}_{2}$ to either of the $\mathrm{Ce}_{2} \mathrm{O}_{3}{ }^{+}$low lying isomers. Bidentate addition of $\mathrm{O}_{2}$ to the $\mathrm{Ce}_{2} \mathrm{O}_{3}{ }^{+}$global minimum lies $2.9 \mathrm{kcal} / \mathrm{mol}$ higher in energy while monodentate addition lies $16.8 \mathrm{kcal} / \mathrm{mol}$ higher. NBO shows that with monodentate addition the closest $\mathrm{O}$ is not covalently bound to $\mathrm{Ce}$. Interestingly the furthest $\mathrm{O}$ has an O-Ce antibond with an bond order of 0.26 . The $\mathrm{O}_{2}$ unit lies between the superoxide unit typically formed with bidentate $\mathrm{O}_{2}$ addition and free $\mathrm{O}_{2}$. Each $\mathrm{O}$ has two lone pairs, which is the case for $\mathrm{O}_{2}$, but the $\mathrm{O}-\mathrm{O}$ bond has an bond order of 1.77 . This value lies between the common value of 1.5 for superoxide and 2.0 for $\mathrm{O}_{2}$.

Placing one $\mathrm{O}$ on each $\mathrm{Ce}$ of the $\mathrm{Ce}_{2} \mathrm{O}_{3}{ }^{+}$global minimum increases the energy to 50.4 $\mathrm{kcal} / \mathrm{mol}$ above the global minimum. Two isomers were found at this energy. NBO shows that one isomer consists of a van der Waals complex of $\mathrm{CeO}_{2}{ }^{0.8}$ and $\mathrm{CeO}_{3}{ }^{0.2}$. The other isomer is 
joined by partial bonds from two $\mathrm{O}$ atoms on the $\mathrm{CeO}_{3}$ side to $\mathrm{Ce}$ on $\mathrm{CeO}_{2}$ side. The partially filled Ce-Ce bond found in $\mathrm{Ce}_{2} \mathrm{O}_{3}{ }^{+}$no longer exists for any of the $\mathrm{Ce}_{2} \mathrm{O}_{5}{ }^{+}$isomers.

Optimized Cartesian coordinates in $\AA$ for lowest doublet and quartet isomers of $\mathrm{Ce}_{2} \mathrm{O}_{5}{ }^{+}$ calculated at the BP86/TZVP level.

a doublet

$\begin{array}{lrrr}\mathrm{O} & 0.219659 & -0.000960 & 2.986703 \\ \mathrm{Ce} & -0.836206 & -0.002155 & 1.543006 \\ \mathrm{O} & 0.106473 & 1.265224 & -0.007177 \\ \mathrm{O} & 0.112963 & -1.263851 & -0.007793 \\ \mathrm{Ce} & 0.965764 & 0.003097 & -1.297012 \\ \mathrm{O} & -0.284933 & 0.660920 & -3.135760 \\ \mathrm{O} & -0.283721 & -0.662274 & -3.133609\end{array}$

b doublet

Ce $\quad-0.680965 \quad 0.212842 \quad 1.280999$

O $\quad-1.920974 \quad 0.481491 \quad-0.546176$

$\begin{array}{llll}\mathrm{O} & 0.146382 & 1.771293 & 0.163941\end{array}$

O $\quad 0.351699 \quad-0.678363 \quad-0.472984$

$\mathrm{Ce} \quad-0.281436 \quad 0.819380 \quad-1.650079$

$\begin{array}{llll}\mathrm{O} & 0.225744 & 0.669129 & 3.394990\end{array}$

O $\quad-0.953263 \quad 1.253034 \quad 3.361730$

c doublet

$\begin{array}{llll}\text { Ce } & 0.394779 & -0.366339 & 1.377437\end{array}$

$\begin{array}{llll}\mathrm{O} & -1.355532 & -0.270013 & 0.162129\end{array}$

$\begin{array}{llll}\mathrm{O} & 0.684923 & 1.248023 & 0.047789\end{array}$

$\begin{array}{llll}\mathrm{O} & 0.866265 & -1.192125 & -0.700213\end{array}$

$\begin{array}{llll}\mathrm{Ce} & -0.251210 & 0.225406 & -1.502894\end{array}$

$\begin{array}{llll}\mathrm{O} & 0.024897 & 0.006842 & 3.576792\end{array}$

$\begin{array}{llll}\mathrm{O} & -0.364457 & 0.348207 & 4.738952\end{array}$

d doublet

$\begin{array}{lrrr}\mathrm{Ce} & 0.360213 & 0.034487 & 1.462361 \\ \mathrm{O} & -1.635618 & -0.212498 & -0.132042 \\ \mathrm{O} & 0.578694 & 1.315241 & 0.054828 \\ \mathrm{O} & 0.874238 & -1.173097 & 0.078579 \\ \mathrm{Ce} & -0.493513 & -0.063272 & -1.642673 \\ \mathrm{O} & 0.303586 & 0.066141 & -3.276974 \\ \mathrm{O} & 0.012067 & 0.032999 & 3.255749\end{array}$

e doublet 


$\begin{array}{lrrr}\mathrm{O} & 0.486940 & -0.020197 & 3.298789 \\ \mathrm{Ce} & 0.042960 & 0.507413 & 1.610996 \\ \mathrm{O} & -0.597160 & 1.323482 & 0.018802 \\ \mathrm{O} & -0.177316 & -1.334749 & 0.001166 \\ \mathrm{Ce} & 0.666652 & -0.385293 & -1.422804 \\ \mathrm{O} & 1.834330 & 0.156934 & -0.007698 \\ \mathrm{O} & 0.535572 & -0.222686 & -3.237082\end{array}$

a quartet

$\begin{array}{lrrr}\mathrm{O} & 0.343267 & 0.168163 & 3.145884 \\ \mathrm{Ce} & -0.857939 & -0.050917 & 1.819328 \\ \mathrm{O} & 0.011823 & 1.114516 & 0.125086 \\ \mathrm{O} & 0.128177 & -1.467288 & 0.411153 \\ \mathrm{Ce} & 1.095775 & -0.251767 & -0.855223 \\ \mathrm{O} & -0.724955 & 0.521863 & -4.252737 \\ \mathrm{O} & 0.003851 & -0.034571 & -3.445133\end{array}$

b quartet

$\begin{array}{lrrr}\mathrm{O} & 1.712841 & 0.633032 & 0.604096 \\ \mathrm{Ce} & 0.234733 & 0.181026 & 1.544332 \\ \mathrm{O} & -1.398996 & 0.895986 & 0.219403 \\ \mathrm{O} & -0.555550 & -1.554983 & 0.400357 \\ \mathrm{Ce} & -1.778145 & -0.697366 & -0.935732 \\ \mathrm{O} & 1.447778 & 0.522238 & -2.390056 \\ \mathrm{O} & 0.337337 & 0.020066 & -2.494045\end{array}$

c quartet

$\begin{array}{lrrr}\mathrm{Ce} & 0.688694 & -0.578092 & 1.092345 \\ \mathrm{O} & -1.251826 & -0.325322 & 0.368422 \\ \mathrm{O} & 0.799604 & 1.212819 & 0.013493 \\ \mathrm{O} & 0.803844 & -1.196391 & -0.943845 \\ \mathrm{Ce} & -0.447904 & 0.369712 & -1.456002 \\ \mathrm{O} & 0.102692 & 0.020708 & 3.916254 \\ \mathrm{O} & -0.695439 & 0.496568 & 4.709325\end{array}$

d quartet

$\begin{array}{lrrr}\mathrm{Ce} & 0.328220 & 0.029901 & 1.590812 \\ \mathrm{O} & -1.534248 & -0.177485 & -0.016911 \\ \mathrm{O} & 0.800594 & 1.217188 & -0.060906 \\ \mathrm{O} & 1.062236 & -0.999209 & -0.060851 \\ \mathrm{Ce} & -0.492688 & -0.051195 & -1.626989 \\ \mathrm{O} & 0.105428 & 0.004171 & -3.358422 \\ \mathrm{O} & -0.269875 & -0.023371 & 3.333095\end{array}$

e quartet
O $\quad 0.361769$
0.137460
3.347663 


$\begin{array}{lrrr}\text { Ce } & 0.037329 & 0.515182 & 1.585731 \\ \text { O } & -0.523784 & 1.235353 & -0.104741 \\ \text { O } & 0.052767 & -1.413753 & 0.159498 \\ \text { Ce } & 0.661778 & -0.380712 & -1.546886 \\ \text { O } & 1.848855 & -0.085545 & 0.139942 \\ \text { O } & 0.353264 & 0.016919 & -3.319037\end{array}$




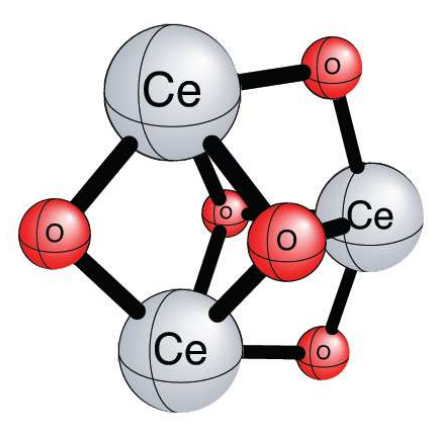

3-5a

$(0.0)$

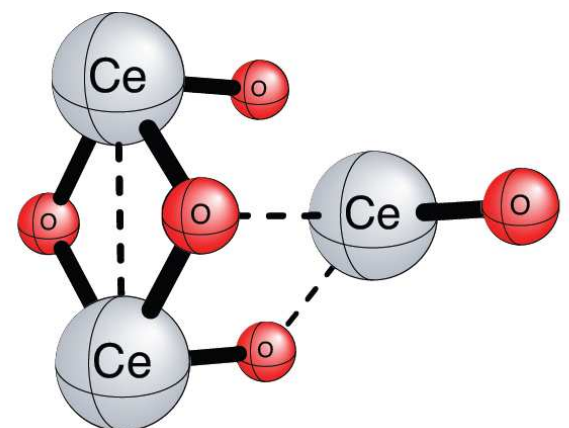

3-5b

(5.6)

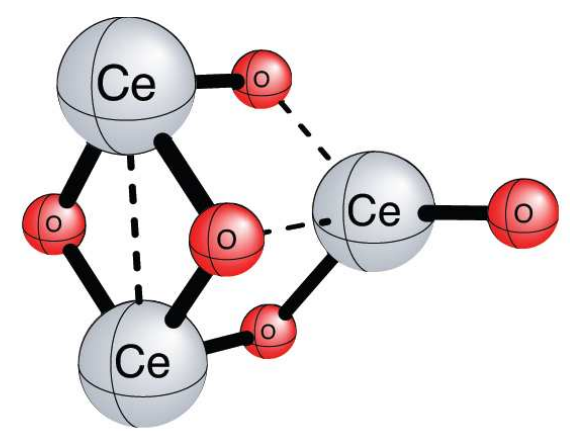

3-5c

(5.7)

Figure S3. Three lowest isomers of $\mathrm{Ce}_{3} \mathrm{O}_{5}{ }^{+}$calculated at the BP86/TZVP level.

Like the ground state of $\mathrm{Ce}_{2} \mathrm{O}_{3}{ }^{+}$, the ground state has pseudo $\mathrm{D}_{3 h}$ symmetry. The three $\mathrm{Ce}$ atoms and three $\mathrm{O}$ atoms alternate in a plane. The $\mathrm{O}-\mathrm{Ce}-\mathrm{O}$ angles are about $141.6^{\circ}$ and the Ce-O-Ce angles are about $98.3^{\circ}$. For the remaining two $\mathrm{O}$ atoms, one lies centered above the plane and the other lies centered below the plane. Each out of plane $\mathrm{O}$ has a single bond to each Ce.

Also like the ground state of $\mathrm{Ce}_{2} \mathrm{O}_{3}{ }^{+}$, the first low lying isomer, $5.6 \mathrm{kcal} / \mathrm{mol}$, is the result of rotating one $\mathrm{O}$ atom around a $\mathrm{Ce}$ atom. One of the out of plane $\mathrm{O}$ atoms is rotated so that both out of plane $\mathrm{O}$ atoms are on the same side of the plane. The rotation pushes the three planar $\mathrm{O}$ atoms below the plane and the remaining $\mathrm{O}$ towards the other $\mathrm{Ce}$ atoms. NBO shows that a half filled $\mathrm{Ce}-\mathrm{Ce}$ bond is formed between the $\mathrm{Ce}$ atoms that are not attached to the rotated $\mathrm{O}$ atom despite the Ce-Ce distance increasing from $3.19 \AA$ to $3.37 \AA$. The bond order for two of the O atoms connected to the $\mathrm{Ce}$ atom with the rotated $\mathrm{O}$ atom is reduced to a half. The third $\mathrm{Ce}-\mathrm{O}$ bond is removed completely. A nearly degenerate isomer, $5.7 \mathrm{kcal} / \mathrm{mol}$, partially fills the missing Ce-O bond and increases one half filled $\mathrm{Ce}-\mathrm{O}$ bond by one. This isomer also has a half filled Ce-Ce bond in the same position. 
Optimized Cartesian coordinates in $\AA$ for lowest doublet and quartet isomers of $\mathrm{Ce}_{3} \mathrm{O}_{5}{ }^{+}$ calculated at the BP86/TZVP level.

a doublet

$\begin{array}{lrrr}\mathrm{O} & 1.994265 & 0.002925 & 1.152077 \\ \mathrm{O} & -0.000276 & 1.300854 & -0.005053 \\ \mathrm{Ce} & 0.000167 & 0.000843 & 1.842496 \\ \mathrm{Ce} & 1.598465 & -0.000195 & -0.922627 \\ \mathrm{O} & -0.000794 & -1.300713 & 0.004610 \\ \mathrm{O} & -1.995209 & 0.003907 & 1.152398 \\ \mathrm{Ce} & -1.598384 & -0.000534 & -0.920628 \\ \mathrm{O} & 0.001313 & -0.007248 & -2.300443\end{array}$

b doublet

$\begin{array}{lrrr}\mathrm{O} & -1.786341 & 0.420192 & -1.011790 \\ \mathrm{O} & 0.641331 & -0.719289 & -0.579335 \\ \mathrm{Ce} & -0.018052 & 1.324688 & -1.024487 \\ \mathrm{Ce} & 1.099106 & -0.549594 & 1.548377 \\ \mathrm{O} & -1.420209 & -3.207843 & -1.459471 \\ \mathrm{Ce} & -1.568097 & -1.760882 & -0.380647 \\ \mathrm{O} & 0.691269 & 1.435393 & 0.954069 \\ \mathrm{O} & -0.628465 & -1.520959 & 1.681934\end{array}$

c doublet

$\begin{array}{lrrr}\mathrm{O} & 1.366525 & 1.147280 & -1.159048 \\ \mathrm{O} & 3.578534 & -1.335537 & 0.459093 \\ \mathrm{Ce} & 2.127225 & -0.908606 & -0.532867 \\ \mathrm{Ce} & -0.039672 & 1.776898 & 0.094554 \\ \mathrm{O} & 0.205923 & -2.132386 & -0.575287 \\ \mathrm{O} & -1.713741 & 0.514301 & -0.097602 \\ \mathrm{Ce} & -1.133517 & -1.364185 & 0.668772 \\ \mathrm{O} & 0.551012 & -0.052565 & 1.142384\end{array}$

d doublet

$\begin{array}{lrrr}\mathrm{O} & 0.000052 & 0.006695 & 2.237327 \\ \mathrm{Ce} & 0.000127 & -0.000941 & 0.418118 \\ \mathrm{Ce} & -0.000098 & 3.295012 & -0.532170 \\ \mathrm{Ce} & -0.000083 & -3.296693 & -0.535942 \\ \mathrm{O} & 1.314119 & 1.919198 & -0.260468 \\ \mathrm{O} & -1.314125 & 1.919018 & -0.260527 \\ \mathrm{O} & -1.314057 & -1.921060 & -0.260643 \\ \mathrm{O} & 1.314067 & -1.921227 & -0.260588\end{array}$

a quartet

O $\quad 2.047677 \quad 0.002665 \quad 1.181425$ 


$\begin{array}{lrrr}\mathrm{O} & -0.000021 & 1.289863 & -0.003076 \\ \mathrm{Ce} & -0.000110 & 0.003138 & 1.849536 \\ \mathrm{Ce} & 1.597325 & -0.001604 & -0.924916 \\ \mathrm{O} & -0.000481 & -1.289674 & 0.004381 \\ \mathrm{O} & -2.048332 & 0.003303 & 1.181646 \\ \mathrm{Ce} & -1.597325 & -0.001440 & -0.923498 \\ \mathrm{O} & 0.000813 & -0.006412 & -2.362667\end{array}$

b quartet

$\begin{array}{lrrr}\mathrm{O} & -1.857655 & 0.430296 & -0.966965 \\ \mathrm{O} & 0.641335 & -0.638024 & -0.712122 \\ \mathrm{Ce} & 0.032838 & 1.482504 & -0.904599 \\ \mathrm{Ce} & 1.113759 & -0.680013 & 1.461846 \\ \mathrm{O} & -1.333845 & -3.446569 & -1.084649 \\ \mathrm{Ce} & -1.523088 & -1.612470 & -0.574539 \\ \mathrm{O} & 0.733202 & 1.374179 & 1.059117 \\ \mathrm{O} & -0.796004 & -1.488199 & 1.450560\end{array}$

c quartet

$\begin{array}{lrrr}\mathrm{O} & 1.350577 & 1.104990 & -1.234113 \\ \mathrm{O} & 3.485198 & -1.710151 & 0.442331 \\ \mathrm{Ce} & 2.142229 & -0.688416 & -0.464737 \\ \mathrm{Ce} & -0.204548 & 1.814394 & 0.088561 \\ \mathrm{O} & 0.406690 & -1.977157 & -0.623232 \\ \mathrm{O} & -1.759944 & 0.423011 & -0.105160 \\ \mathrm{Ce} & -1.049797 & -1.396450 & 0.715225 \\ \mathrm{O} & 0.571884 & 0.074979 & 1.181125\end{array}$

d quartet

$\begin{array}{lrrr}\mathrm{O} & -0.010101 & -0.001426 & 2.397932 \\ \mathrm{Ce} & -0.001585 & 0.000309 & 0.255575 \\ \mathrm{Ce} & 0.003002 & 3.282980 & -0.501925 \\ \mathrm{Ce} & 0.003052 & -3.282759 & -0.499469 \\ \mathrm{O} & 1.313701 & 1.827253 & -0.273197 \\ \mathrm{O} & -1.310764 & 1.828051 & -0.281120 \\ \mathrm{O} & -1.310892 & -1.828188 & -0.280985 \\ \mathrm{O} & 1.313589 & -1.826217 & -0.271705\end{array}$




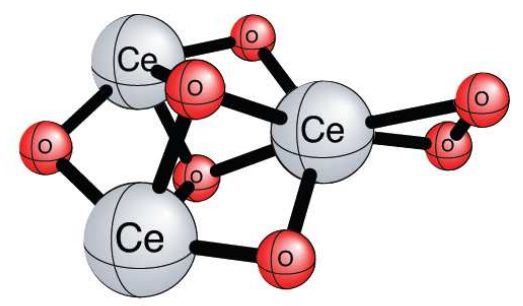

3-7a

$(0.0)$

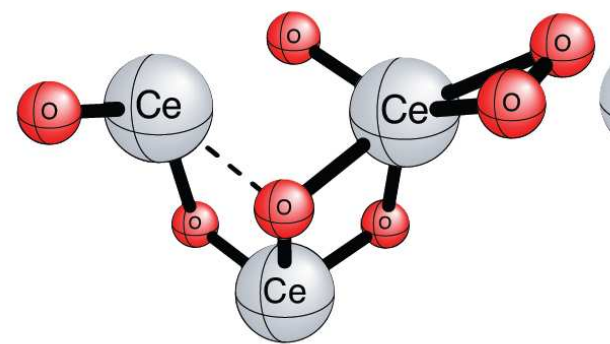

3-7b

(5.5)

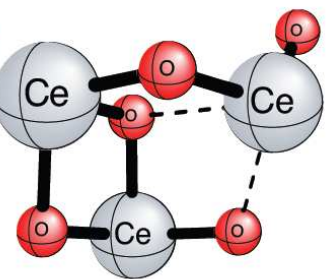

3-7c (30.1)

Figure S4. Three lowest isomers of $\mathrm{Ce}_{3} \mathrm{O}_{7}{ }^{+}$calculated at the BP86/TZVP level.

The global minimum is formed with the addition of $\mathrm{O}_{2}$ to the $\mathrm{Ce}_{3} \mathrm{O}_{5}{ }^{+}$global minimum. NBO shows that $\mathrm{O}_{2}$ adds in a bidentate fashion to one Ce but otherwise does not significantly change the bonding scheme of the molecule. Like $\mathrm{Ce}_{3} \mathrm{O}_{5}{ }^{+}$, the first low lying isomer, 5.5 $\mathrm{kcal} / \mathrm{mol}$, is the result of an out of plane $\mathrm{O}$ becoming attached to only one Ce through a rotation to the other side of the plane. A higher lying excited state, $30.1 \mathrm{kcal} / \mathrm{mol}$, shows similarities to the $\mathrm{Ce}_{2} \mathrm{O}_{5}{ }^{+}$monodentate addition, each $\mathrm{O}$ in the free $\mathrm{O}_{2}$ unit has two lone pairs and the $\mathrm{O}-\mathrm{O}$ bond order has been reduced. In this case, the bond order has been reduced further to 1.40 .

Optimized Cartesian coordinates in $\AA$ for lowest doublet and quartet isomers of $\mathrm{Ce}_{3} \mathrm{O}_{7}{ }^{+}$ calculated at the BP86/TZVP level.

a doublet

$\begin{array}{lrrr}\mathrm{O} & 2.020029 & 0.000561 & 1.003620 \\ \mathrm{O} & 0.000892 & 1.279190 & -0.102764 \\ \mathrm{Ce} & 0.003065 & 0.001382 & 1.860268 \\ \mathrm{Ce} & 1.586267 & -0.001719 & -0.977525 \\ \mathrm{O} & 0.000559 & -1.280086 & -0.099304 \\ \mathrm{O} & -2.015820 & 0.001062 & 1.009211 \\ \mathrm{Ce} & -1.586355 & -0.001409 & -0.972283 \\ \mathrm{O} & -0.001213 & -0.003488 & -2.363469 \\ \mathrm{O} & -0.003839 & 0.660189 & 4.122378 \\ \mathrm{O} & -0.004036 & -0.655841 & 4.122699\end{array}$

b doublet 


$\begin{array}{lrrr}\mathrm{O} & 1.348648 & 0.864588 & -1.560943 \\ \mathrm{O} & 3.506624 & -1.197835 & 0.687474 \\ \mathrm{Ce} & 2.166543 & -0.950591 & -0.491348 \\ \mathrm{Ce} & -0.139669 & 1.757911 & -0.570318 \\ \mathrm{O} & 0.219900 & -2.233231 & -0.248933 \\ \mathrm{O} & -1.781262 & 0.312277 & -0.308400 \\ \mathrm{Ce} & -1.075890 & -1.228420 & 0.801574 \\ \mathrm{O} & 0.523367 & 0.146571 & 0.890812 \\ \mathrm{O} & 0.305622 & 3.482203 & 0.942655 \\ \mathrm{O} & -0.131596 & 4.091728 & -0.142572\end{array}$

c doublet

$\begin{array}{lrrr}\mathrm{O} & 1.617181 & 1.180193 & -0.835926 \\ \mathrm{O} & 3.433004 & -0.835063 & 1.527364 \\ \mathrm{Ce} & 2.374750 & -0.797617 & 0.062035 \\ \mathrm{Ce} & -0.099125 & 1.727312 & -0.050763 \\ \mathrm{O} & 0.574890 & -2.194578 & -0.319134 \\ \mathrm{O} & -1.550188 & 0.314029 & -0.636517 \\ \mathrm{Ce} & -1.088805 & -1.454541 & 0.411399 \\ \mathrm{O} & 0.257937 & 0.025816 & 1.230508 \\ \mathrm{O} & 3.575034 & -1.629711 & -2.108115 \\ \mathrm{O} & 3.247606 & -1.890643 & -3.280854\end{array}$

d doublet

$\begin{array}{lrrr}\mathrm{O} & 2.120111 & 0.151193 & 0.938093 \\ \mathrm{O} & -0.035557 & 1.440006 & -0.010353 \\ \mathrm{Ce} & 0.109119 & 0.584252 & 1.841660 \\ \mathrm{Ce} & 1.630862 & -0.214142 & -0.965054 \\ \mathrm{O} & -0.023140 & -1.181959 & 0.047639 \\ \mathrm{O} & -2.131335 & 0.010875 & 0.923713 \\ \mathrm{Ce} & -1.724442 & -0.153821 & -0.927967 \\ \mathrm{O} & 0.126017 & -0.047513 & -2.261185 \\ \mathrm{O} & -0.112092 & 0.090942 & 3.617204 \\ \mathrm{O} & -2.574308 & -0.026926 & -2.536613\end{array}$

e doublet

$\begin{array}{lrrr}\mathrm{O} & 1.471918 & 0.815394 & -1.493174 \\ \mathrm{O} & 3.360484 & -1.692073 & 0.726259 \\ \mathrm{Ce} & 2.136461 & -0.885540 & -0.421456 \\ \mathrm{Ce} & 0.087068 & 1.941583 & -0.312585 \\ \mathrm{O} & 0.310226 & -1.892618 & -0.340704 \\ \mathrm{O} & -1.705462 & 0.507371 & -0.192397 \\ \mathrm{Ce} & -1.188160 & -1.036701 & 0.955379 \\ \mathrm{O} & 0.703302 & 0.460098 & 1.024772 \\ \mathrm{O} & -1.196367 & -2.181270 & 2.409786 \\ \mathrm{O} & -0.699471 & 3.621163 & -0.529050\end{array}$


f doublet

$\begin{array}{lrrr}\mathrm{O} & 1.685639 & 0.875910 & -1.321159 \\ \mathrm{O} & 2.984603 & -0.411245 & 1.771620 \\ \mathrm{Ce} & 2.483935 & -0.862121 & 0.070002 \\ \mathrm{Ce} & 0.021948 & 1.732773 & -0.680366 \\ \mathrm{O} & 0.710188 & -2.255802 & -0.109315 \\ \mathrm{O} & -1.406548 & -0.009734 & -0.970739 \\ \mathrm{Ce} & -0.981785 & -1.355868 & 0.402414 \\ \mathrm{O} & 0.388221 & 0.162591 & 0.927119 \\ \mathrm{O} & 2.924511 & -1.526965 & -1.557185 \\ \mathrm{O} & -1.234504 & 3.009361 & -0.189912\end{array}$

a quartet

$\begin{array}{lrrr}\mathrm{O} & 2.189221 & 0.023371 & 0.586550 \\ \mathrm{O} & 0.094645 & 1.298539 & -0.409428 \\ \mathrm{Ce} & 0.302143 & 0.150116 & 1.533481 \\ \mathrm{Ce} & 1.523186 & -0.120146 & -1.411436 \\ \mathrm{O} & 0.025281 & -1.293387 & -0.189011 \\ \mathrm{O} & -1.766565 & 0.171088 & 1.105462 \\ \mathrm{Ce} & -1.639873 & -0.016235 & -0.994241 \\ \mathrm{O} & -0.232945 & -0.179062 & -2.570329 \\ \mathrm{O} & -0.605652 & -0.061158 & 5.469317 \\ \mathrm{O} & 0.110104 & 0.026717 & 4.482468\end{array}$

b quartet

$\begin{array}{lrrr}\mathrm{O} & 0.977153 & 1.053531 & -1.123307 \\ \mathrm{O} & 3.596751 & -0.799837 & 0.576399 \\ \mathrm{Ce} & 2.117990 & -0.803738 & -0.467731 \\ \mathrm{Ce} & -0.598711 & 1.343437 & 0.054673 \\ \mathrm{O} & 0.519245 & -2.422629 & -0.640080 \\ \mathrm{O} & -1.956879 & -0.252857 & -0.268659 \\ \mathrm{Ce} & -1.038906 & -1.975012 & 0.507738 \\ \mathrm{O} & 0.301816 & -0.352657 & 1.108490 \\ \mathrm{O} & 0.084068 & 4.211936 & 0.258848 \\ \mathrm{O} & 0.939760 & 5.043028 & -0.006371\end{array}$

c quartet

$\begin{array}{lrrr}\mathrm{O} & 1.648174 & 1.156565 & -0.804130 \\ \mathrm{O} & 3.429163 & -0.825825 & 1.661317 \\ \mathrm{Ce} & 2.344182 & -0.759553 & 0.210811 \\ \mathrm{Ce} & -0.110859 & 1.730165 & -0.080564 \\ \mathrm{O} & 0.621606 & -2.177779 & -0.290361 \\ \mathrm{O} & -1.544151 & 0.301047 & -0.675591 \\ \mathrm{Ce} & -1.093954 & -1.458414 & 0.397050 \\ \mathrm{O} & 0.249543 & 0.036434 & 1.244744\end{array}$




$\begin{array}{llll}\mathrm{O} & 3.558666 & -1.612735 & -2.265341 \\ \mathrm{O} & 3.239913 & -1.944708 & -3.397938\end{array}$

d quartet

$\begin{array}{lrrr}\mathrm{O} & 3.145334 & -0.400125 & -0.421853 \\ \mathrm{O} & 1.044586 & 1.525417 & 0.942176 \\ \mathrm{Ce} & -0.326088 & 0.614603 & 2.015376 \\ \mathrm{Ce} & 1.480212 & 0.215698 & -0.961130 \\ \mathrm{O} & -0.135229 & -0.793635 & 0.256373 \\ \mathrm{O} & -2.159881 & 0.940760 & 0.583302 \\ \mathrm{Ce} & -1.988735 & -0.302194 & -0.931084 \\ \mathrm{O} & -0.127406 & 0.679824 & -1.990207 \\ \mathrm{O} & -1.237440 & -0.229178 & 3.392877 \\ \mathrm{O} & -2.310118 & -1.598262 & -2.218693\end{array}$

e quartet

$\begin{array}{lrrr}\mathrm{O} & 1.437573 & 0.824746 & -1.474859 \\ \mathrm{O} & 3.268165 & -1.764837 & 0.675923 \\ \mathrm{Ce} & 2.152353 & -0.758368 & -0.417765 \\ \mathrm{Ce} & -0.066851 & 1.942120 & -0.244902 \\ \mathrm{O} & 0.346698 & -1.882062 & -0.310739 \\ \mathrm{O} & -1.723064 & 0.454453 & -0.177350 \\ \mathrm{Ce} & -1.192519 & -1.157752 & 0.955802 \\ \mathrm{O} & 0.643743 & 0.517453 & 1.058295 \\ \mathrm{O} & -1.122006 & -2.227547 & 2.475849 \\ \mathrm{O} & -0.464093 & 3.709201 & -0.713424\end{array}$

f quartet

$\begin{array}{lrrr}\mathrm{O} & 1.607323 & 0.805171 & -1.285344 \\ \mathrm{O} & 2.382788 & -0.163073 & 1.517529 \\ \mathrm{Ce} & 2.616724 & -1.024458 & -0.286489 \\ \mathrm{Ce} & 0.034574 & 1.755742 & -0.535787 \\ \mathrm{O} & 0.704902 & -2.190869 & -0.192533 \\ \mathrm{O} & -1.435562 & 0.039447 & -0.796160 \\ \mathrm{Ce} & -0.970677 & -1.378819 & 0.493616 \\ \mathrm{O} & 0.228395 & 0.231528 & 1.180957 \\ \mathrm{O} & 3.544395 & -1.851953 & -1.649861 \\ \mathrm{O} & -1.136655 & 3.136184 & -0.103450\end{array}$




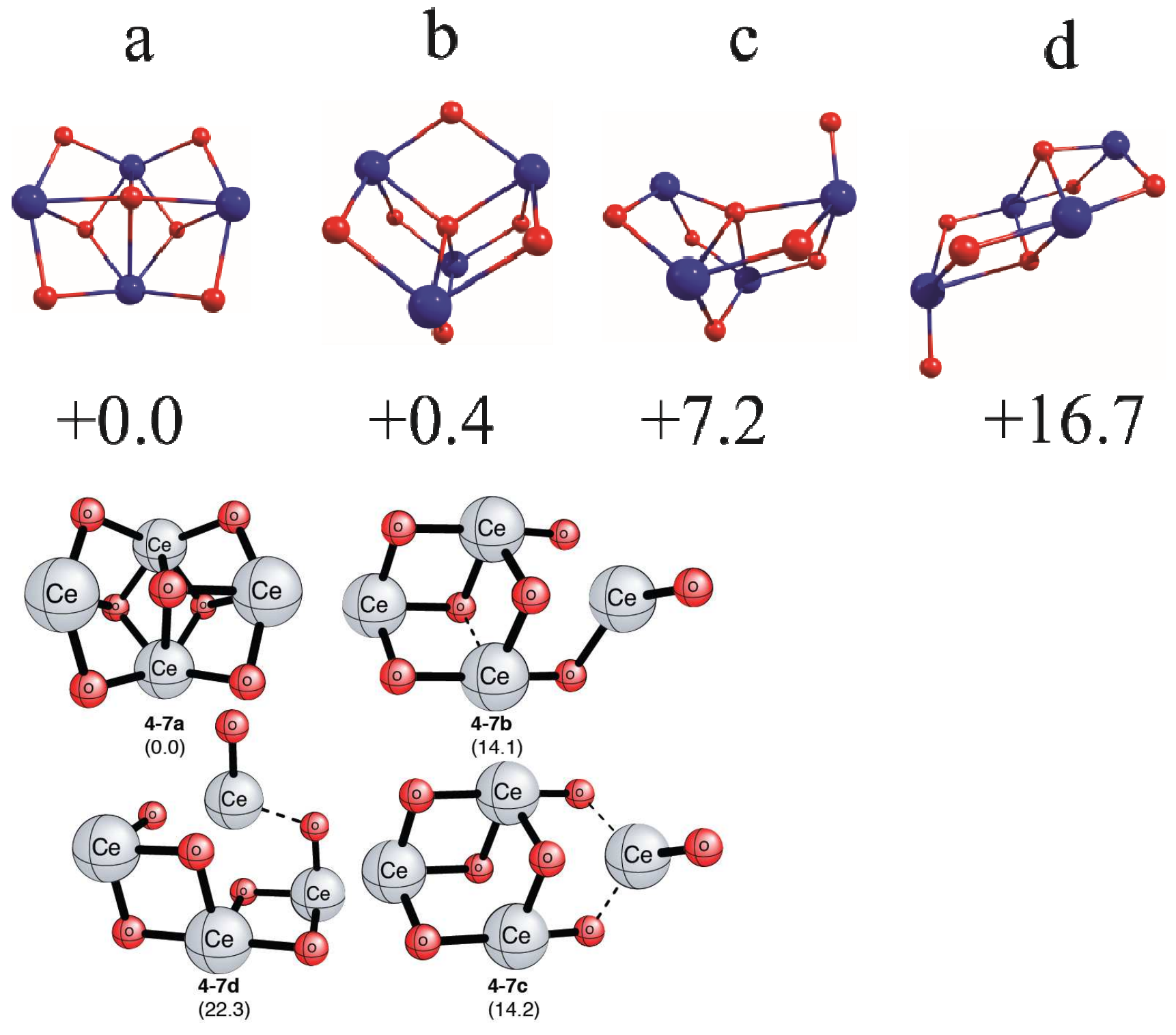

Figure S5. Four lowest doublet and quartet isomers of $\mathrm{Ce}_{4} \mathrm{O}_{7}{ }^{+}$calculated at the BP86/TZVP level.

The global minimum has a puckered ring formed from alternating four $\mathrm{Ce}$ atoms and four $\mathrm{O}$ atoms. Two $\mathrm{O}$ atoms are below the ring and one $\mathrm{O}$ atom is above the ring, where each $\mathrm{O}$ atom is bonded to three $\mathrm{Ce}$ atoms. Another cage structure is shown in isomer b, lying only 0.4 $\mathrm{kcal} / \mathrm{mol}$ higher in energy. Swinging one oxygen out of the cage to make a terminal oxygen bond give isomer c, which is $7.2 \mathrm{kcal} / \mathrm{mol}$ higher in energy. Similar to $\mathrm{Ce}_{2} \mathrm{O}_{3}{ }^{+}$and $\mathrm{Ce}_{3} \mathrm{O}_{5}{ }^{+}$the first low 
lying isomer, $16.7 \mathrm{kcal} / \mathrm{mol}$, is the result of swinging an out of plane $\mathrm{O}$ towards a Ce. The newly formed $\mathrm{CeO}$ is only connected to the rest of the structure by a single $\mathrm{Ce}-\mathrm{O}$ bond.

Optimized Cartesian coordinates in $\AA$ for lowest doublet and quartet isomers of $\mathrm{Ce}_{4} \mathrm{O}_{7}^{+}$ calculated at the BP86/TZVP level.

a doublet

$\begin{array}{lrrr}\mathrm{O} & 0.335557 & 0.284399 & -1.254171 \\ \mathrm{O} & 1.194877 & 0.835098 & 1.251616 \\ \mathrm{Ce} & -0.856287 & 0.893877 & 0.335534 \\ \mathrm{Ce} & 1.666968 & -1.076980 & -0.254854 \\ \mathrm{O} & -0.343482 & -1.242122 & 0.800341 \\ \mathrm{O} & -1.275012 & 0.688664 & 2.359482 \\ \mathrm{O} & 3.520181 & -0.083625 & 0.198207 \\ \mathrm{Ce} & 3.197149 & 0.404616 & 2.189252 \\ \mathrm{O} & 2.103860 & -1.538574 & 1.907930 \\ \mathrm{Ce} & 0.234281 & -0.736033 & 2.919809 \\ \mathrm{O} & 1.895111 & 0.291800 & 3.810631\end{array}$

b doublet

$\begin{array}{lrrr}\mathrm{O} & -1.923045 & -1.631995 & 0.153961 \\ \mathrm{Ce} & -0.200474 & -1.845629 & 1.423481 \\ \mathrm{Ce} & -1.773941 & 0.158106 & -1.022246 \\ \mathrm{O} & 0.003098 & 0.059378 & -2.226612 \\ \mathrm{O} & 1.562946 & -1.926726 & 0.194170 \\ \mathrm{O} & -0.033892 & -0.039681 & 0.244692 \\ \mathrm{Ce} & 1.745200 & -0.142911 & -0.984159 \\ \mathrm{Ce} & 0.098998 & 1.669836 & 1.563267 \\ \mathrm{O} & -1.626120 & 1.850191 & 0.291907 \\ \mathrm{O} & 1.857524 & 1.549803 & 0.333656 \\ \mathrm{O} & -0.063416 & -0.137362 & 2.716913\end{array}$

c doublet

$\begin{array}{lrrr}\mathrm{O} & -1.962215 & -1.687989 & -0.124520 \\ \mathrm{Ce} & -0.192942 & -2.404753 & 1.072450 \\ \mathrm{Ce} & -1.778501 & 0.195176 & -0.841533 \\ \mathrm{O} & -0.065319 & 0.129244 & -2.058428 \\ \mathrm{O} & 1.577745 & -2.048030 & -0.276623 \\ \mathrm{O} & 0.026631 & -0.084194 & 0.681848 \\ \mathrm{Ce} & 1.722366 & -0.157865 & -0.980981 \\ \mathrm{Ce} & 0.258439 & 1.986243 & 1.346845 \\ \mathrm{O} & -1.467702 & 2.077750 & 0.245712 \\ \mathrm{O} & 1.876725 & 1.742982 & 0.107866\end{array}$




\begin{tabular}{|c|c|c|c|}
\hline $\mathrm{O}$ & -0.055996 & -2.173929 & 2.882038 \\
\hline \multicolumn{4}{|c|}{ d doublet } \\
\hline $\mathrm{O}$ & -1.511171 & -1.892099 & -3.358716 \\
\hline $\mathrm{Ce}$ & -2.655016 & -2.785091 & -1.895329 \\
\hline $\mathrm{Ce}$ & 0.438752 & -1.404011 & -2.500889 \\
\hline $\mathrm{O}$ & -0.706943 & -2.603622 & -0.901566 \\
\hline $\mathrm{O}$ & -3.051321 & -1.413901 & -0.408465 \\
\hline $\mathrm{O}$ & -0.059450 & 0.093357 & -0.999436 \\
\hline $\mathrm{Ce}$ & -1.168109 & -0.913186 & 0.585732 \\
\hline $\mathrm{Ce}$ & 2.096268 & 0.307666 & 0.089902 \\
\hline $\mathrm{O}$ & 2.242122 & -1.1411270 & -1.656145 \\
\hline $\mathrm{O}$ & 2.380146 & 2.096107 & -0.042092 \\
\hline $\mathrm{O}$ & 0.594607 & -0.635019 & 1.509889 \\
\hline
\end{tabular}

a quartet

$\begin{array}{lrrr}\mathrm{O} & -1.497375 & -1.804544 & -3.394924 \\ \mathrm{Ce} & -2.667814 & -2.800337 & -2.026601 \\ \mathrm{Ce} & 0.421822 & -1.322892 & -2.435362 \\ \mathrm{O} & -0.699139 & -2.616046 & -0.944439 \\ \mathrm{O} & -3.117830 & -1.546403 & -0.443593 \\ \mathrm{O} & -0.187980 & 0.130527 & -0.872596 \\ \mathrm{Ce} & -1.262394 & -1.070389 & 0.605292 \\ \mathrm{Ce} & 1.914773 & 0.394551 & 0.216549 \\ \mathrm{O} & 2.238505 & -0.988599 & -1.363374 \\ \mathrm{O} & 2.698411 & 1.962711 & -0.482074 \\ \mathrm{O} & 0.758906 & -0.629504 & 1.564005\end{array}$

b quartet

$\begin{array}{lrrr}\mathrm{O} & -2.526861 & 1.375626 & 1.031609 \\ \mathrm{Ce} & -1.865450 & -0.513321 & 1.903701 \\ \mathrm{O} & 0.320313 & -0.175711 & 2.122870 \\ \mathrm{Ce} & -1.695083 & 1.563284 & -0.854541 \\ \mathrm{O} & 0.362457 & 1.699947 & -0.693389 \\ \mathrm{Ce} & 1.090359 & -0.208882 & 0.068644 \\ \mathrm{O} & -1.107504 & -0.518070 & -0.272205 \\ \mathrm{Ce} & 0.954075 & -2.336890 & 2.867303 \\ \mathrm{O} & -1.161868 & -2.400320 & 2.564702 \\ \mathrm{O} & 1.736585 & -2.201878 & 0.982070 \\ \mathrm{O} & 1.056400 & -2.297905 & 4.752432\end{array}$

c quartet

$\begin{array}{lrrr}\mathrm{O} & 0.323027 & 0.225743 & -1.316795 \\ \mathrm{O} & 1.216278 & 0.930284 & 1.242822 \\ \mathrm{Ce} & -0.800390 & 0.989639 & 0.335237 \\ \mathrm{Ce} & 1.689035 & -1.060753 & -0.325611\end{array}$




$\begin{array}{lrrr}\mathrm{O} & -0.259336 & -1.271173 & 0.833881 \\ \mathrm{O} & -1.323885 & 0.617683 & 2.388346 \\ \mathrm{O} & 3.549401 & -0.172272 & 0.173024 \\ \mathrm{Ce} & 3.173517 & 0.483422 & 2.179651 \\ \mathrm{O} & 2.018356 & -1.547710 & 1.881960 \\ \mathrm{Ce} & 0.213891 & -0.711118 & 2.989997 \\ \mathrm{O} & 1.873308 & 0.237370 & 3.881264\end{array}$

d quartet

$\begin{array}{lcrr}\mathrm{O} & -2.440768 & 1.513449 & 0.979870 \\ \mathrm{Ce} & -1.738253 & -0.252833 & 2.055449 \\ \mathrm{O} & 0.476094 & -0.330795 & 2.171634 \\ \mathrm{Ce} & -1.786696 & 1.347718 & -0.983799 \\ \mathrm{O} & 0.215398 & 1.439195 & -1.122045 \\ \mathrm{Ce} & 1.177942 & -0.325229 & -0.079959 \\ \mathrm{O} & -1.199966 & -0.577592 & -0.103799 \\ \mathrm{Ce} & 0.752487 & -2.497690 & 2.597612 \\ \mathrm{O} & -1.299720 & -2.276677 & 2.719084 \\ \mathrm{O} & 1.297260 & -2.393020 & 0.592821 \\ \mathrm{O} & 2.895753 & 0.549921 & 0.006833\end{array}$




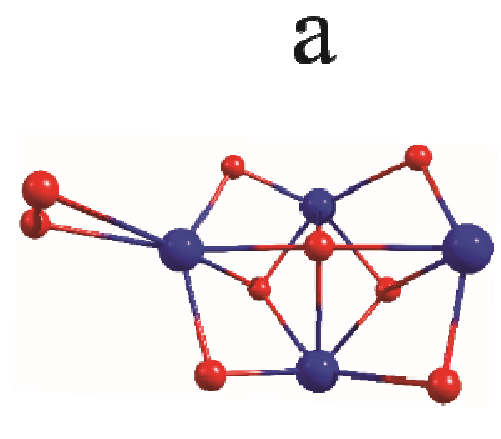

b

C
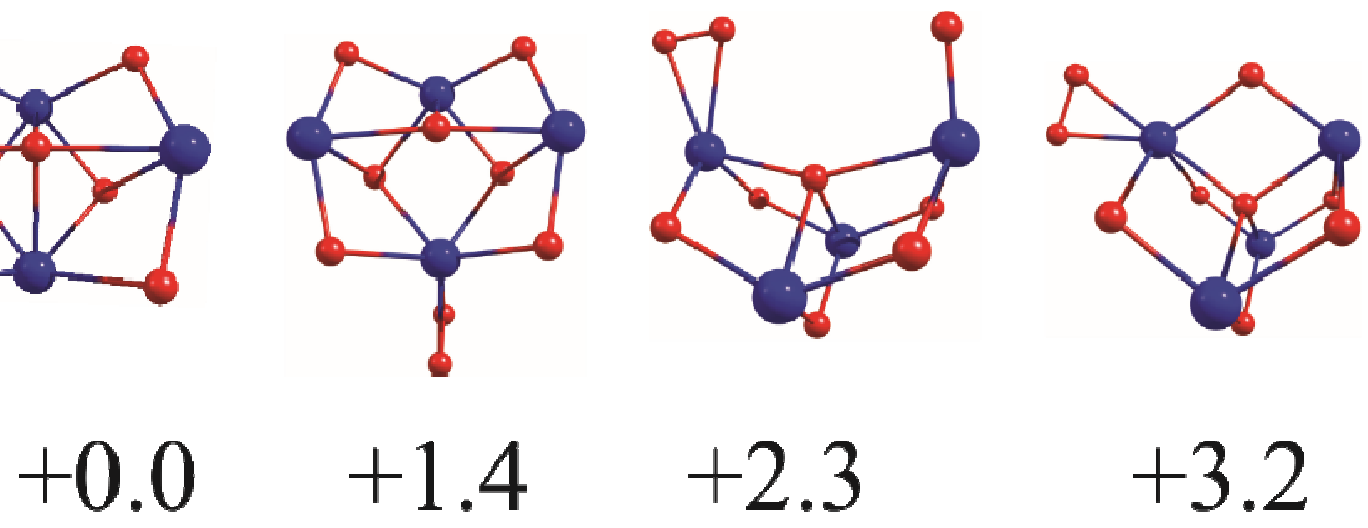

$+3.2$

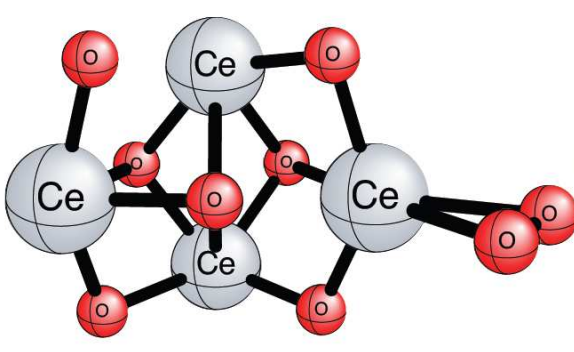

4-9a

$(0.0)$

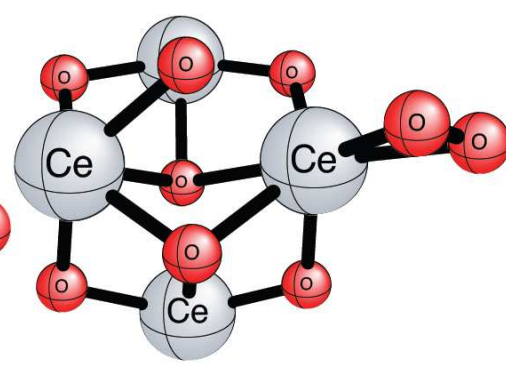

4-9b

(1.9)

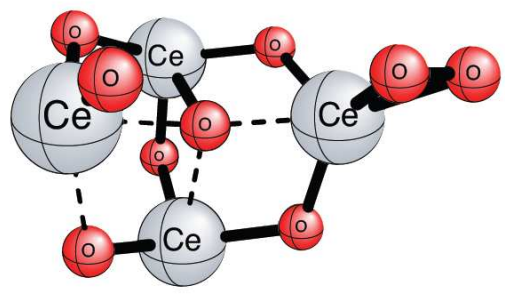

4-9c

(3.7)

Figure S6. Four lowest doublet and three lowest quartet isomers of $\mathrm{Ce}_{4} \mathrm{O}_{9}{ }^{+}$calculated at the BP86/TZVP level.

The global minimum is formed with the addition of $\mathrm{O}_{2}$ to the $\mathrm{Ce}_{4} \mathrm{O}_{7}^{+}$global minimum. Moving $\mathrm{O}_{2}$ to the next $\mathrm{Ce}$ atom only increased the energy by $1.9 \mathrm{kcal} / \mathrm{mol}$. Several structures can be formed by moving $\mathrm{O}_{2}$ around the first low lying isomer of $\mathrm{Ce}_{4} \mathrm{O}_{7}^{+}$. Addition of $\mathrm{O}_{2}$ to other $\mathrm{Ce}_{4} \mathrm{O}_{7}{ }^{+}$isomers $(4,7 \mathrm{c}$ and 4,7b) produces additional low-lying isomers 4,9c and 4,9d, which are only 2.3 and $3.2 \mathrm{kcal} / \mathrm{mol}$ higher, respectively.

Optimized Cartesian coordinates in $\AA$ for lowest doublet and quartet isomers of $\mathrm{Ce}_{4} \mathrm{O}_{9}{ }^{+}$ calculated at the BP86/TZVP level. 
a doublet

$\begin{array}{lrrr}\mathrm{O} & 0.541330 & -0.489473 & -1.297240 \\ \mathrm{O} & 1.032830 & 0.602796 & 1.117614 \\ \mathrm{Ce} & -0.997368 & 0.057648 & 0.103762 \\ \mathrm{Ce} & 2.012586 & -1.280064 & -0.095259 \\ \mathrm{O} & 0.148188 & -1.852061 & 0.951722 \\ \mathrm{O} & -1.335297 & -0.056741 & 2.230267 \\ \mathrm{O} & 3.539525 & 0.230870 & 0.264929 \\ \mathrm{Ce} & 2.985347 & 0.884284 & 2.123040 \\ \mathrm{O} & 2.479166 & -1.301867 & 2.125536 \\ \mathrm{Ce} & 0.397466 & -0.904567 & 2.938731 \\ \mathrm{O} & 1.709278 & 0.656801 & 3.705650 \\ \mathrm{O} & -3.014982 & 0.505844 & -1.051072 \\ \mathrm{O} & -2.424867 & 1.667653 & -0.853902\end{array}$

b doublet

$\begin{array}{lrrr}\mathrm{O} & 0.163247 & -0.245419 & -1.033252 \\ \mathrm{O} & 1.355956 & 0.973801 & 1.040139 \\ \mathrm{Ce} & -0.761084 & 0.986484 & 0.372900 \\ \mathrm{Ce} & 1.409756 & -1.404900 & 0.252477 \\ \mathrm{O} & -0.405864 & -0.940520 & 1.415252 \\ \mathrm{O} & -1.007541 & 1.463997 & 2.316167 \\ \mathrm{O} & 3.367560 & -0.563645 & 0.152208 \\ \mathrm{Ce} & 3.361969 & 0.573769 & 1.900666 \\ \mathrm{O} & 2.090585 & -1.189867 & 2.338360 \\ \mathrm{Ce} & 0.416910 & 0.103948 & 3.336177 \\ \mathrm{O} & 2.369845 & 1.126685 & 3.567492 \\ \mathrm{O} & -0.249824 & -1.644665 & 4.770643 \\ \mathrm{O} & -0.438308 & -0.518548 & 5.434546\end{array}$

c doublet

$\begin{array}{lrrr}\mathrm{O} & -2.124066 & -1.406252 & 0.063022 \\ \mathrm{Ce} & -0.427417 & -1.990049 & 1.444044 \\ \mathrm{Ce} & -1.765960 & 0.370982 & -0.893460 \\ \mathrm{O} & 0.039182 & 0.239595 & -2.054663 \\ \mathrm{O} & 1.371086 & -2.032142 & 0.128706 \\ \mathrm{O} & -0.080487 & -0.060307 & 0.389349 \\ \mathrm{Ce} & 1.711722 & -0.220100 & -0.800704 \\ \mathrm{Ce} & 0.136286 & 1.513326 & 1.851310 \\ \mathrm{O} & -1.508076 & 1.943357 & 0.550945 \\ \mathrm{O} & 1.920039 & 1.336699 & 0.663930 \\ \mathrm{O} & -0.248427 & -0.254561 & 2.839025 \\ \mathrm{O} & 0.348512 & -3.582422 & 3.011145 \\ \mathrm{O} & -0.773909 & -4.061818 & 2.512005\end{array}$


d doublet

$\begin{array}{lrrr}\mathrm{O} & -2.566124 & -0.455839 & 0.645550 \\ \mathrm{Ce} & -1.727764 & 0.059283 & 2.552311 \\ \mathrm{O} & -0.244258 & 1.446497 & 1.993639 \\ \mathrm{Ce} & -1.136029 & -1.253097 & -0.645067 \\ \mathrm{O} & 0.469665 & 0.055571 & -0.901177 \\ \mathrm{Ce} & 1.379472 & 0.581306 & 0.967518 \\ \mathrm{O} & -0.122459 & -1.170248 & 1.368879 \\ \mathrm{Ce} & 0.987227 & -2.195712 & 3.210333 \\ \mathrm{O} & -0.863074 & -1.086789 & 3.942336 \\ \mathrm{O} & 2.300411 & -0.556668 & 2.330336 \\ \mathrm{O} & 0.957926 & -3.907455 & 2.618985 \\ \mathrm{O} & -0.818054 & -3.567710 & -0.747439 \\ \mathrm{O} & -1.453518 & -3.263259 & -1.863012\end{array}$

\begin{tabular}{lrrr}
\multicolumn{4}{l}{ a quartet } \\
$\mathrm{O}$ & 0.505112 & -1.873704 & -1.153962 \\
$\mathrm{O}$ & 0.154555 & -0.052380 & 0.823429 \\
$\mathrm{Ce}$ & -0.914231 & -1.993744 & 0.371439 \\
$\mathrm{Ce}$ & 2.202903 & -1.148078 & -0.063993 \\
$\mathrm{O}$ & 1.033823 & -2.461511 & 1.379375 \\
$\mathrm{O}$ & -1.321756 & -1.719960 & 2.381236 \\
$\mathrm{O}$ & 2.386380 & 0.956619 & -0.350818 \\
$\mathrm{Ce}$ & 1.506720 & 1.653046 & 1.416817 \\
$\mathrm{O}$ & 2.493286 & -0.271254 & 2.002587 \\
$\mathrm{Ce}$ & 0.599947 & -0.993635 & 3.052181 \\
$\mathrm{O}$ & 0.586664 & 1.137698 & 3.221252 \\
$\mathrm{O}$ & -1.531751 & 2.519338 & -0.752148 \\
$\mathrm{O}$ & -0.628449 & 2.968681 & -0.063624
\end{tabular}

b quartet

$\begin{array}{lrrr}\mathrm{O} & 0.104054 & 0.084198 & -1.185176 \\ \mathrm{O} & 1.378030 & 0.962353 & 1.039824 \\ \mathrm{Ce} & -0.810972 & 0.956233 & 0.461208 \\ \mathrm{Ce} & 1.566531 & -1.155499 & -0.198050 \\ \mathrm{O} & -0.240119 & -1.104642 & 1.164671 \\ \mathrm{O} & -0.892874 & 1.078388 & 2.531712 \\ \mathrm{O} & 3.495128 & -0.192054 & -0.193196 \\ \mathrm{Ce} & 3.507166 & 0.603999 & 1.725105 \\ \mathrm{O} & 2.357725 & -1.312993 & 1.927575 \\ \mathrm{Ce} & 0.682345 & -0.294238 & 3.067686 \\ \mathrm{O} & 2.481449 & 0.811585 & 3.516170 \\ \mathrm{O} & -0.598611 & -1.054489 & 5.565895 \\ \mathrm{O} & -1.356648 & -0.661723 & 6.440350\end{array}$




\begin{tabular}{lrrr}
\multicolumn{4}{l}{ c quartet } \\
$\mathrm{O}$ & -2.590518 & -0.009416 & 0.628711 \\
$\mathrm{Ce}$ & -1.695858 & 0.120184 & 2.635266 \\
$\mathrm{O}$ & -0.113025 & 1.470342 & 2.306447 \\
$\mathrm{Ce}$ & -1.204766 & -0.686051 & -0.722402 \\
$\mathrm{O}$ & 0.463613 & 0.502133 & -0.837994 \\
$\mathrm{Ce}$ & 1.434257 & 0.654803 & 1.128869 \\
$\mathrm{O}$ & -0.212839 & -1.036962 & 1.224293 \\
$\mathrm{Ce}$ & 0.810752 & -2.429415 & 2.850177 \\
$\mathrm{O}$ & -0.900721 & -1.349409 & 3.796881 \\
$\mathrm{O}$ & 2.251518 & -0.826456 & 2.263997 \\
$\mathrm{O}$ & 0.494012 & -3.914585 & 1.803122 \\
$\mathrm{O}$ & -0.991573 & -3.386041 & -1.061899 \\
$\mathrm{O}$ & -0.581430 & -4.423249 & -0.542274
\end{tabular}

